\title{
Assessment of the Impact of the Planetary Scale on the Decay of Blocking and the Use of Phase Diagrams and Enstrophy as a Diagnostic
}

\author{
A. R. Lupo ${ }^{a}$, I. I. Mokhov ${ }^{b}$, S. Dostoglou ${ }^{a}$, A. R. Kunz ${ }^{a}$, and J. P. Burkhardt ${ }^{a}$ \\ ${ }^{a}$ University of Missouri-Columbia, Columbia, MO, USA \\ ${ }^{b}$ Oboukhov Institute of Atmospheric Physics, Russian Academy of Sciences, Pyzhevskii per. 3, Moscow, 119017 Russia \\ e-mail:mokhov@ifaran.ru \\ Received January 13, 2006; in final form, May 31, 2006
}

\begin{abstract}
It was shown that abrupt changes in the large-scale structure of atmospheric flows may lead to the rapid decay of blocking. Analysis of phase diagrams made it possible to identify when sharp changes occurred in the dynamics of the system. The connection of these changes to the decay of blocking was estimated for three blocking events in the Southern Hemisphere. In addition to phase diagrams, enstrophy was used as a diagnostic tool for the analysis of blocking events. From the results of this analysis, four scenarios for the decay mechanisms were determined: (i) decay with a lack of synoptic-scale support, (ii) decay with an active role for synoptic processes, and (iii-iv) either of these mechanisms in the interaction with an abrupt change in the character of the planetary-scale flow.
\end{abstract}

DOI: $10.1134 / \mathrm{S} 0001433807010057$

\section{INTRODUCTION}

In recent decades, atmospheric blocking events (blocks) have been widely studied with the goal of understanding dynamic processes that influence their existence, intensity, and duration [1-7]. Since three earlier publications [11-13], it has been generally thought that blocks are the result of the interaction between an amplifying synoptic-scale wave and a quasi-stationary planetary-scale wave. Blocking regi;mes are maintained by the influx of anticyclonic vorticity into the blocking region via amplification of synoptic-scale waves [1-7]. In these studies, different diagnostic techniques have been used to determine the mechanisms involved in the growth, maintenance, and decay of blocking.

In recent publications that examined blocking in the Northern and Southern hemispheres, atmospheric processes were partitioned into planetary- and synoptic-scale components in order to examine either the relative role of each scale and their interaction or the nature of the interaction itself in both the Northern and Southern hemispheres [1-9]. In the analysis of the variability of atmospheric circulation, atmospheric processes can likewise be partitioned into the highfrequency component, with time scales of 2-6 days, and the low-frequency component, of duration longer than 10 days [10]. As was discussed in [10], the maxima of low-frequency variability, which contributes significantly to the total variability of atmospheric circulation, are located in regions with a maximum frequency of blocking events.
In these and earlier studies [11-15], it has been found that the synoptic scale plays an important role in the lifecycle of atmospheric blocks. In many studies, the impact of the synoptic scale (synoptic forcing) is shown to be large in comparison with the planetaryscale forcing. However, in other studies, it has been concluded that the impact of the planetary scale is very essential to the lifecycle of blocks [16, 17]. Although the studies referenced above do not understate the role of the planetary scale, they focus more on the impact of synoptic-scale contributions.

In a model study [16], it is argued that blocking events will decay when there is a considerable change in the planetary-scale flow regime. In [17], the emphasis is placed on the role of planetary-scale deformation as providing a favorable background for block development (Colucci, 2003; personal communication). Both these studies confirm the point of view that, while planetary-scale processes may not by themselves result in the onset and maintenance of blocks, they are able to create conditions favorable for the interaction with synoptic-scale processes. Thus, in the case of a significant change in the large-scale flow regime, the blocks, with no synoptic support, would be expected to decay quite rapidly.

The goal of this study is to demonstrate how changes in the large-scale structure of atmospheric flows can influence the blocking regime. In order to achieve this goal, the behavior of three Southern Hemisphere blocking events [7-9] were examined. 
Characteristics of the blocking events analyzed in the paper

\begin{tabular}{l|l|c|c}
\hline \multicolumn{1}{|c|}{ Period } & $\begin{array}{c}\text { Duration, } \\
\text { day }\end{array}$ & $\begin{array}{c}\text { Block intensity } \\
\text { (from [43]) }\end{array}$ \\
\hline 1 & July 23-August 2, 1986 & 10.5 & 3.64 \\
2 & August 3-16, 1986 & 13.5 & 4.06 \\
3 & July 13-24, 2001 & 11.0 & 3.33 \\
\hline
\end{tabular}

\section{DATA AND ANALYSIS METHODS}

The data set used in the paper was the NCEP/NCAR reanalysis [18] at 6-h intervals on a $2.5^{\circ} \times 2.5^{\circ}$ latitude-longitude grid at 17 mandatory levels from 1000 to $10 \mathrm{hPa}$. These data include standard atmospheric variables, such as geopotential height, temperature, relative humidity, and vertical and horizontal wind components, as well as surface information. The data at mandatory levels were interpolated quadratically in $\ln p$, where $p$ is pressure, to isobaric surfaces in 50-hPa increments [19].

To determine the times of block formation and decay, the blocking criterion proposed in [20] was used (see also the references in the Introduction). This criterion is a modification of the blocking index [45]. Characteristics of the selected blocking events in the Southern Hemisphere over the Pacific Ocean are listed in the table. A climatological, synoptic, and dynamic description of the three blocking events listed in the table for July-August 1986 and July 2001 with the use of potential vorticity diagnostics can be found in [7, $8]$. The diagnostic technique used in $[7,8]$ is described in more detail in [19]. Potential vorticity was calculated on $300-\mathrm{hPa}$ surfaces since these fields of potential vorticity are similar to those calculated on an isentropic surface [19].

The emphasis in this study was placed on the analysis of phase diagrams, in particular, on their use to demonstrate that changes in the planetary-scale flow regimes could be correlated to block onset and decay. To extract planetary-scale variability, the techniques proposed in [21, 22] (see also [23-25]) or in [26] for extracting interannual variations from time series were used here with modifications. A similar analysis was carried out in [27] with the aim of understanding the behavior of the planetary-scale flow associated with blocking. The techniques referenced above are based on the classical analysis of dynamic systems.

Analysis was performed on time series of the 500-hPa height for July and August 1986 and July 2001. The planetary-scale height fields were averaged over a box of $40^{\circ}$ latitude by $60^{\circ}$ longitude within the blocking region to obtain one number for each time period. The procedure is analogous to that used in [28] to derive the wave amplitude index, except that, in our study, the fields were first filtered and then averaged over a box. In [28], the midlatitude geopotential height field was first averaged and then filtered to produce one number for the time period.

To separate the planetary-scale wavelengths from the synoptic-scale wavelengths, we used a secondorder two-dimensional filter [29]. The filtered data retain 2,44 , and $80 \%$ of the original signal at wavelengths of 3000,4500 , and $6000 \mathrm{~km}$ at $45^{\circ} \mathrm{N}$ (or $45^{\circ} \mathrm{S}$ ). A detailed description of the use of the filtering procedure can be found in [3].

The midlatitude planetary-scale atmospheric flow has cyclic regimes [28, 30-32] associated, in particular, with the propagation of Rossby waves in the atmosphere [33, 34]. However, the planetary-scale flow may have two or more relatively stable states $[1$, 35-37]. In the Northern Hemisphere during the fall, along with stable midlatitude tropospheric westerly flow regimes, there exist quasi-regular anomalies, Indian summer-type blocking events, and these were associated in $[33,38]$ with a stationary Rossby wave. Thus, in this paper, particular attention was given to a diagnosis of changes in the atmospheric flow regime in connection with block onset and decay by the example of individual blocking events.

To determine specific features of the dynamics of block formation and evolution, trajectories in the phase plane with the abscissa $X$ and ordinate $d X / d t$ were analyzed by means of using a time series for the variable (blocking characteristic) $X(t)$ analogously to [21-25]. For regimes that are cyclical, the process can be fitted by a harmonic oscillator. The trajectories for a harmonic oscillator in the phase plane are circular. For stable regimes, trajectories approach an attractor, in particular, a limit cycle. The attractor regime, generally speaking, may change its character. It should be noted that, in general, circular trajectories do not necessarily correspond to the attractor. (A trajectory can also be located around the cycle long enough when the cycle is weakly unstable.)

For the diagnosis of regimes related to blocks, their dynamics, and stability, Lyapunov exponents can be applied also [10], which are widely used in the study of the stability of dynamic systems. As applied to the problem considered, it is possible, for example, to examine local Lyapunov exponents extracted from the barotropic vorticity equation with forcing [39] (see also [9]). A numerical implementation of this principle in [39] using data for a 3-yr period after application of a 15-day filter (planetary scale) for domains over the North Atlantic and western Europe showed a strong correlation between the sum of positive Lyapunov exponents, eigenvalues of the linearized barotropic vorticity equation, and the domain-integrated enstrophy.

It should be noted that the strict treatment of atmospheric flows as the set of trajectories for an infinite- 


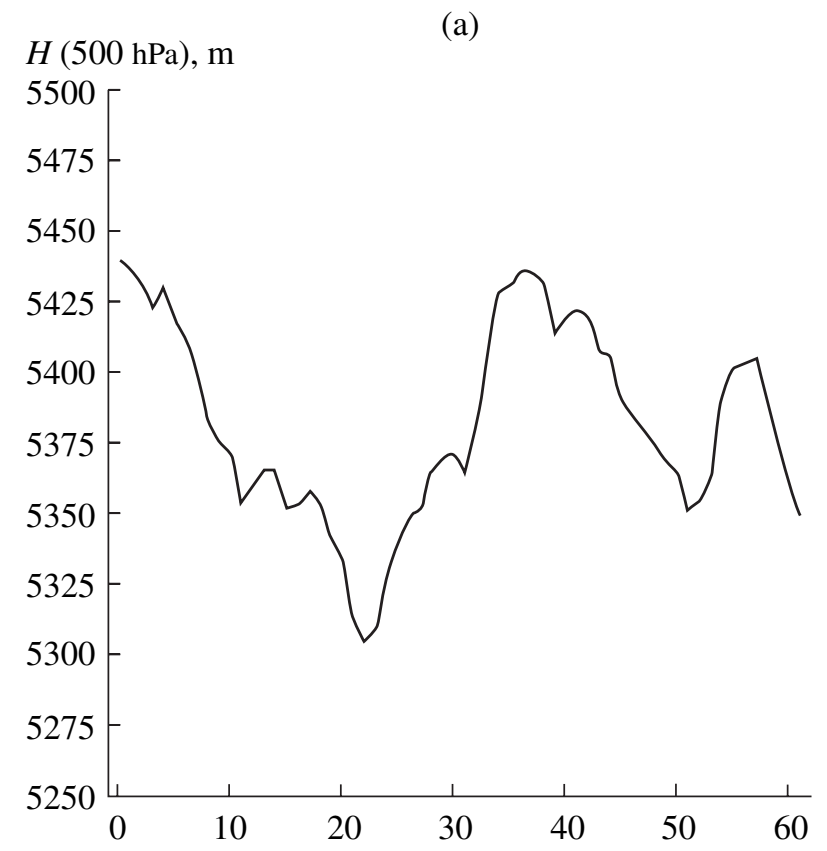

(b)

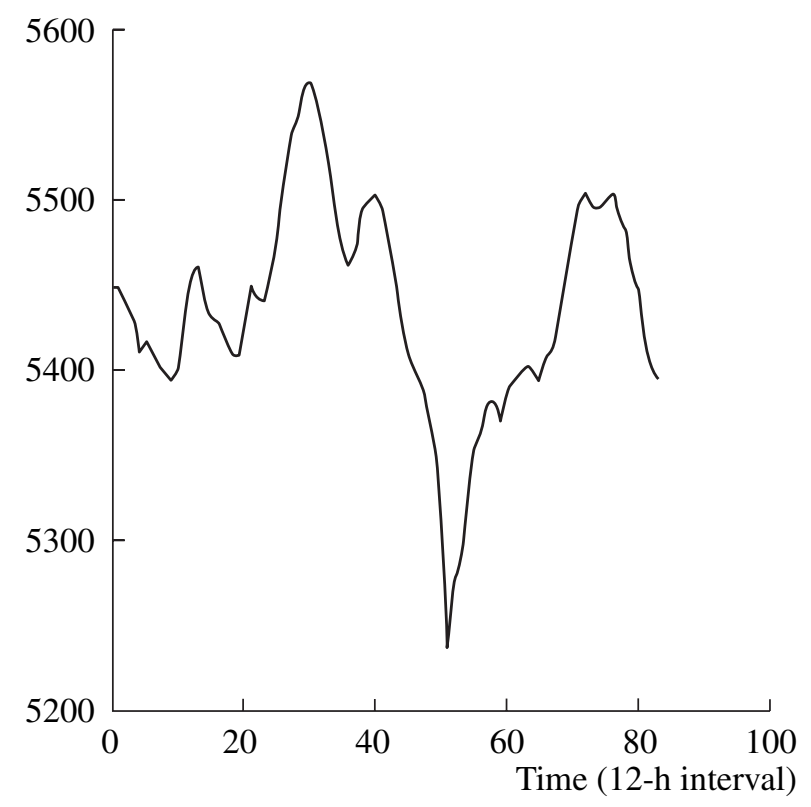

Fig. 1. Dynamics of the mean 500-hPa height (at 12-h intervals) in southeast Pacific blocking regions in (a) July 2001 and (b) July 20 to August 31, 1986.

dimensional system requires the development of the theory for calculating Lyapunov exponents [40-42].

As applied to atmospheric blocking, it must be noted that, prior to the onset of a blocking event, there always exists an upstream ridge accompanied by an amplifying shortwave within one-half wavelength, which is linked to the ridge dynamically [20]. Given that the ridge and the shortwave are related to increasing height gradients, the observation noted above can

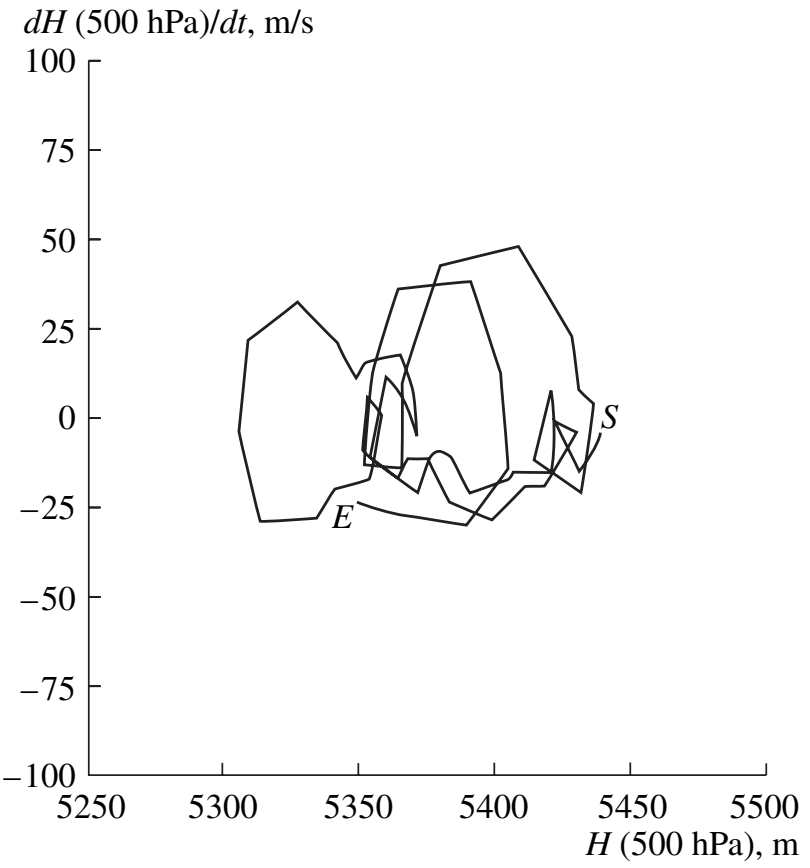

Fig. 2. Phase diagram of the mean $500-\mathrm{hPa}$ height $(\mathrm{m})$ for a box $\left(130^{\circ}\right.$ to $170^{\circ} \mathrm{W}, 30^{\circ}$ to $\left.60^{\circ} \mathrm{S}\right)$ in the Southern Hemisphere midlatitude flow in July 2001 ( $S$ and $E$ mark the start and end points of the phase trajectory).

be interpreted as the criterion for the formation of a regime with sufficient instability to initiate the transition from zonal flow to blocking. Moreover, observational data $[20,43]$ are indicative of a strong correlation between the block intensity and the mean anticyclonic vorticity in the vicinity of a block.

\section{RESULTS OF ANALYSIS}

\section{Blocking Event in the Southeast Pacific in July 2001}

The blocking event chosen for analysis was an event in the southeast Pacific Ocean during July 2001. Figure 1a shows the variation of the mean $500-\mathrm{hPa}$ height over the analyzed region in July 2001 with a 12-h time interval. In July, the mean heights within the blocking region decreased prior to the time of block onset ( 25 half-day periods). The heights rise until the block reaches a maximum development and then fall again. This behavior in the planetary-scale height field suggests changes in the large-scale flow.

Figure 2 displays an example of the phase diagram (trajectory) showing the dynamics of the mean 500 -hPa height in July 2001. It can be seen that the behavior of the large-scale flow is complicated, with periods when the flow is relatively more stable or less stable.

A clearer picture of the flow regimes can be obtained by means of dividing the total trajectory into 
different parts during July 2001. In Fig. 3a, the month is broken up into equal 10-day periods and, within each period, different types of behavior are discernible. Thus, the evolution is stable during the first part of the month (solid curve), while the two consecutive 10-day periods (dotted and dashed curves) in the second part show less stable behavior. If the month is broken up into parts in a different way, for example, when the solid trajectory shows the dynamics until the time of block formation (Fig. 3b), it can be seen that the flow loses stability before the appearance of the block and during its development phase. This type of behavior of the flow in the blocking region is not surprising and was previously noted [12].

The lifecycle of the block is characterized by a trajectory that has a near-circular path but then becomes unstable, after which a new cycle with a nearly circular trajectory begins. After block dissipation, a spiral trajectory emerges. On the phase diagram, the cyclic behavior with a shift can be seen, thus suggesting a different, unstable, regime of the planetary-scale flow.

\section{Blocking Events in the Southeast Pacific in July-August 1986}

Figure 1b, like Fig. 1a, shows the change in the mean 500-hPa height in the blocking region over the southeastern part of the Pacific Ocean in the second half of July and during August 1986. The height values initially remain constant until late July and then grow rapidly in early August (time interval of approximately 25 days) as soon as a second block appears. It is shown in [7] that the heights in the blocking region are greater for the second block. This means that the second event is stronger (table). Then, the heights fall rapidly, and the blocking regime (two simultaneously occurring blocks) breaks down abruptly around August 18, 1986.

In addition, phase trajectories can be used to diagnose the blocking regime of July-August 1986 (Fig. 4), when three blocks occurred in the Pacific Ocean basin between July 20 and August 18. Two consecutive blocks formed in the southeast Pacific, and a third block developed in the southwestern part in midAugust. In Fig. 5, the first part of the trajectory (solid curve), during the onset time of the block and throughout its lifecycle, suggests a relatively stable flow regime. Subsequently, the flow moves to another state as the second block develops and, during its lifecycle, the trajectory suggests a relatively stable regime (dotted line). At the end of the lifecycle of the second block and after its decay (dashed line), the flow becomes unstable again and the two events, which occurred simultaneously over the southwestern and southeastern parts of the Pacific Ocean, come to an abrupt end during a $24-\mathrm{h}$ period.

Calculations of enstrophy and Lyapunov exponents similar to [39] for July 2001 (Fig. 6a) show that

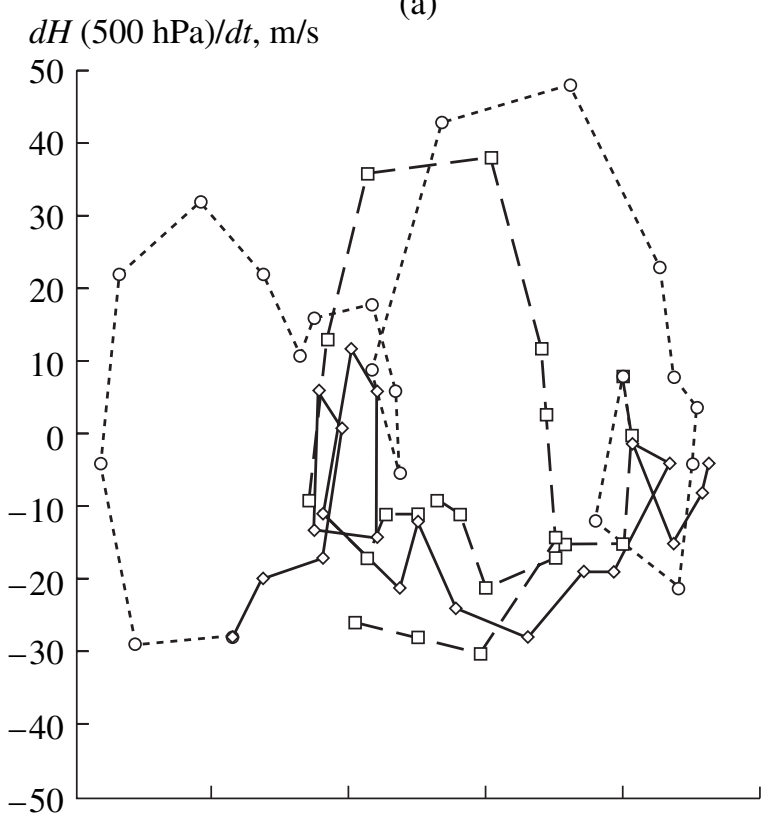

(b)

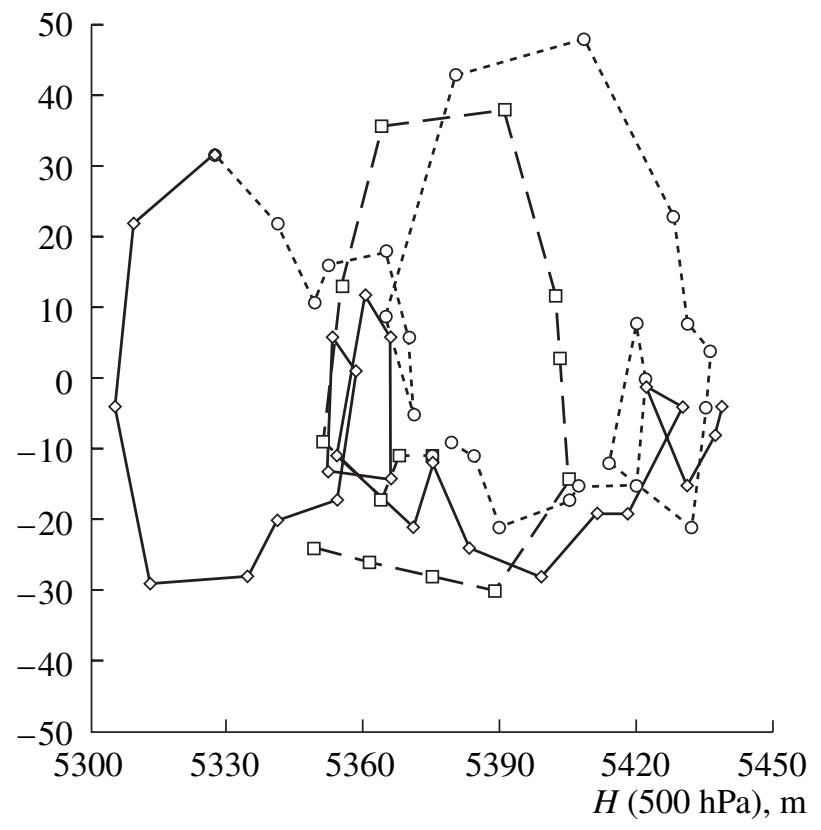

Fig. 3. Phase trajectory for the $500-\mathrm{hPa}$ height in the central part of the blocking region in July 2001 for (a) 10-day periods and (b) before block onset, during block lifecycles, and after block decay.

these values correspond well to Fig. 1a. In particular, the changes displayed in Figs. 6a coincide temporally with those in Fig. 1a (and also in Fig. 3a). In Fig. 6a, the area-averaged enstrophy reaches a maximum shortly after the onset of the block and remains at the level of a relative minimum during the lifecycle of this block. The enstrophy was calculated from the data that were used to construct Fig. 1. This circumstance is also consistent with the fact that, in a quasi-barotropic 


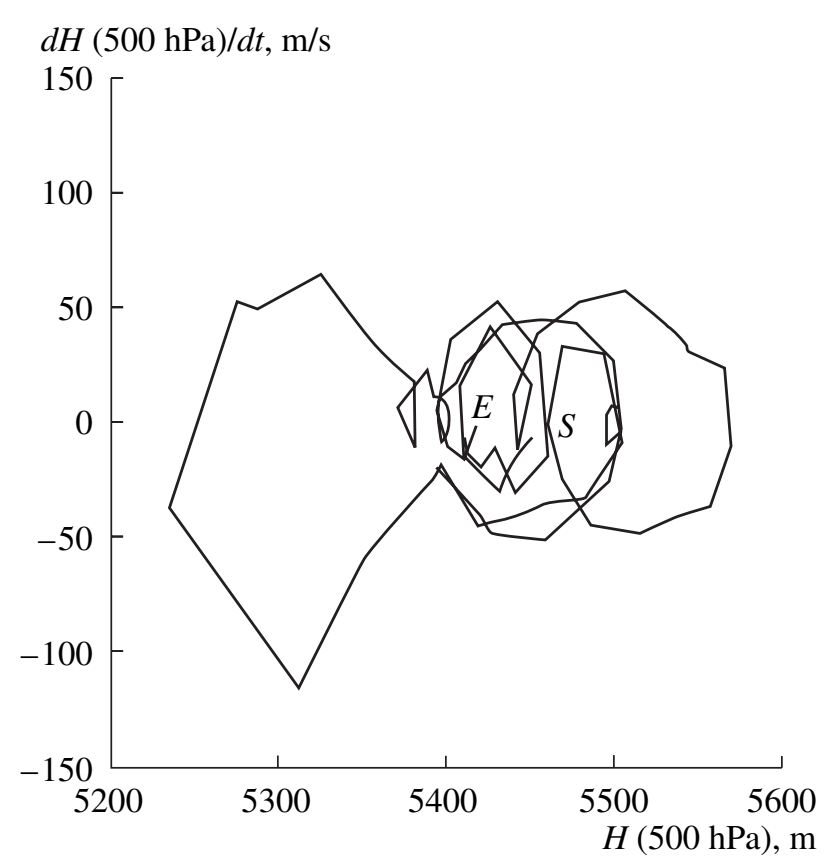

Fig. 4. As in Fig. 2 except for July-August 1986.

flow (the planetary-scale flow should be strongly barotropic), the blocking regime represents a state with the minimum enstrophy (and entropy; see, e.g., [44]). The positive Lyapunov exponents are relatively small during blocking events. This implies that, once the blocking event has formed, the predictability for the large-scale flow increases.

Figure 6b, like Fig. 6a, shows similar changes in the behavior of the planetary-scale flow with the formation of a blocking event in July-August 1986.

\section{DISCUSSION}

For the selected blocking events, it was found that analysis of trajectories on a phase diagram may be useful in examining the nature of the planetary-scale flow regime during the development of blocking events. In July 2001, the flow regime moved from one state to another, relatively less stable, state during and after the decay of the block. During the July and August 1986 events, there were two quasi-steady states representing the successive blocks described in the table. Then, the two simultaneous blocks came to an abrupt end during a less stable period from mid- to late August. The results obtained show that the decay of a block can be correlated with changes in the structure of the planetary-scale flow $[1,16,17]$. This circumstance indicates that the planetary-scale processes are essential in the lifecycles of the blocks since these processes provide a favorable background for blocking, in spite of large contributions of synoptic-scale

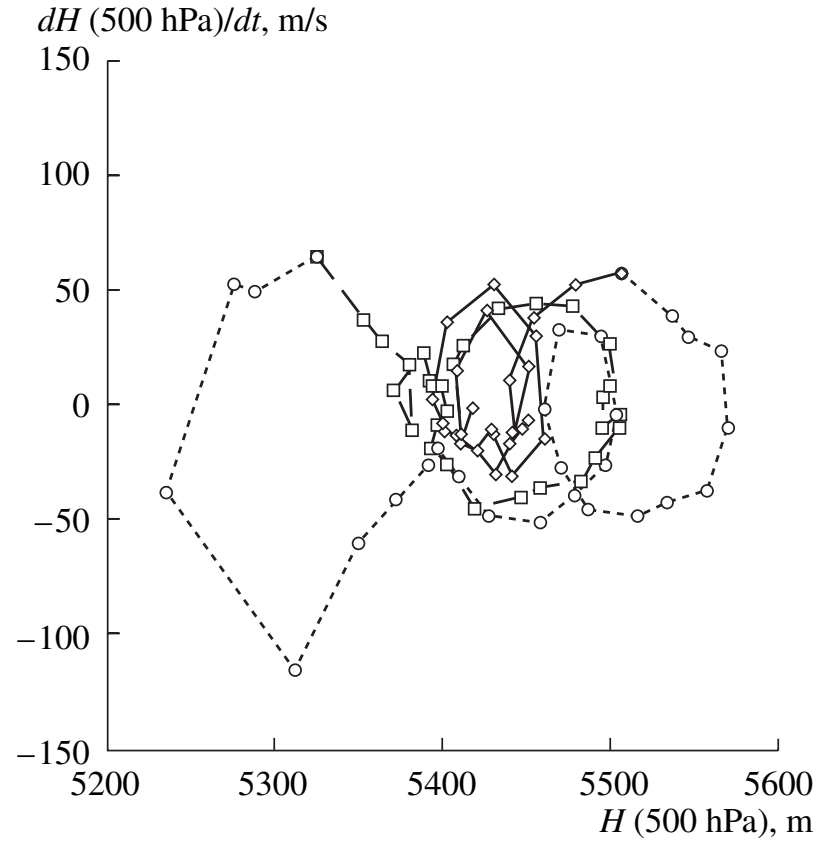

Fig. 5. As in Fig. 3b except for July-August 1986 in the preblock period, during the lifecycles of the first and second blocks, and after their decay.

processes and the interaction of the forcing components.

Additional analysis of the role of the changes in the planetary-scale flow regimes in the lifecycle of blocks was carried out by means of calculating the Lyapunov components as a characteristic of flow stability [39].

As might be expected, the area-integrated enstrophy values (Fig. 5) [39] in the blocking region decrease to a minimum during the lifecycle of the block. This result suggests that the planetary-scale flow becomes less stable around the time of the onset and decay of the July 2001 block. It may be possible that the planetary-scale flow in these periods evolves (geostrophically) from one quasi-steady state to another. The features shown in Figs. 1-3 support this interpretation. In general, it should be noted that relatively simple techniques provide a useful diagnosis of complex nonlinear atmospheric phenomena. They may also be helpful in analyzing the predictability of atmospheric processes; however, more comprehensive studies are required to confirm their applicability.

The rapid decay of the 1986 blocking event demonstrates the influence that the planetary scale exerts on this process. The simultaneous occurrence of the blocks in 1986 is a remarkable example. The results obtained here, however, do not necessarily contrast with the conclusions that the synoptic-scale processes play an important role in the lifecycles of blocking events. Thus, it is shown in [4] that the formation and intensification of two simultaneous blocks was governed by local synoptic-scale processes. Previously in [45], a similar inference was made 


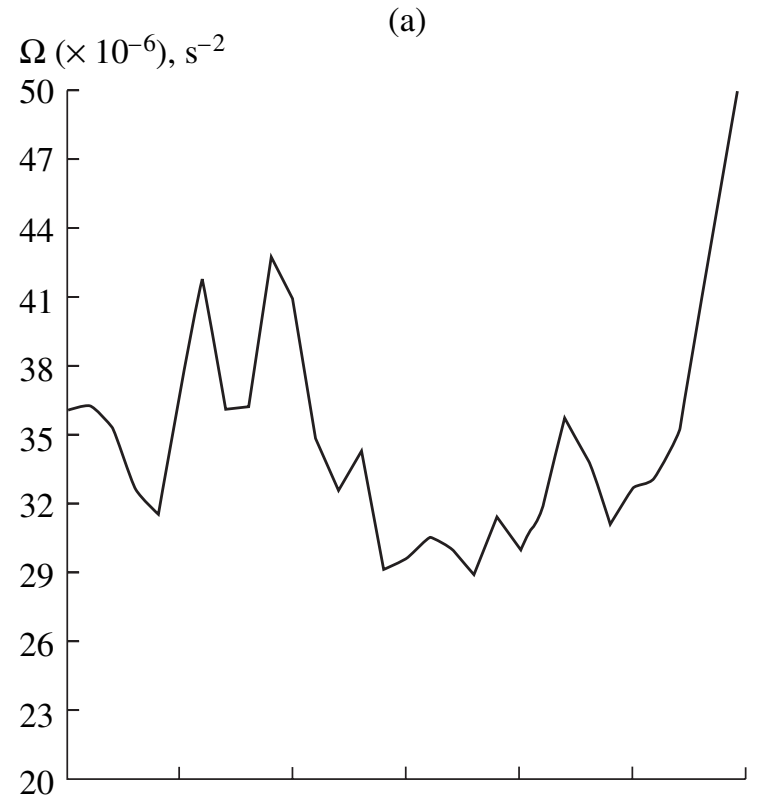

(b)

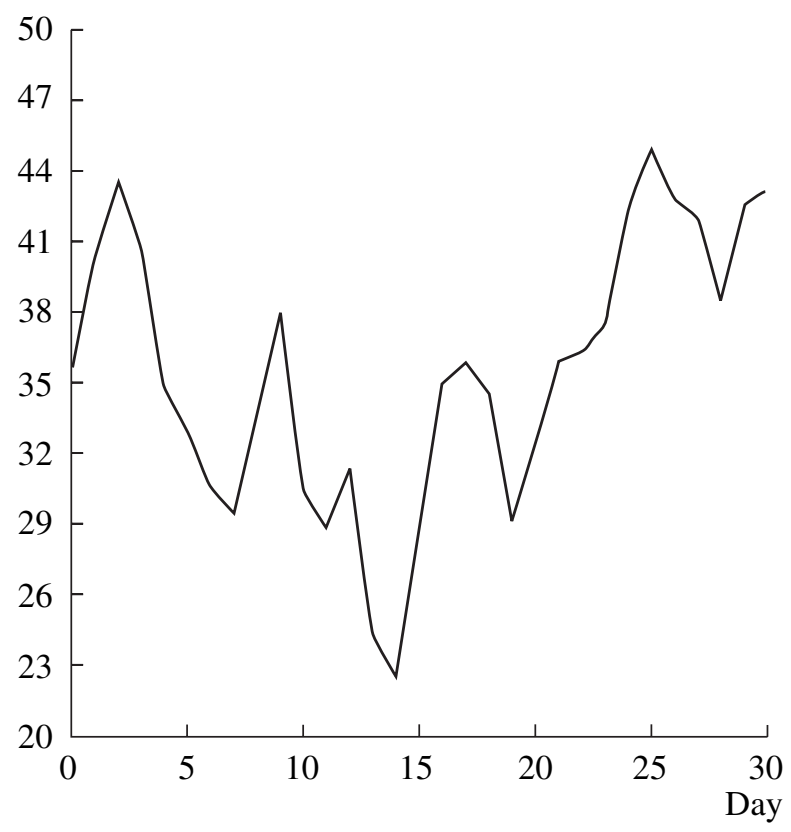

Fig. 6. Area-averaged enstrophy $\Omega$ for the blocking events in (a) 2001 (July 1-31) and (b) 1986 (July 20-August 20).

on the basis of statistical analysis. However, the decay of blocks may occur when there is no longer active synoptic-scale feeding into the blocking region, when synoptic-scale processes have a negative impact on the blocks, or when there is a change in the planetary-scale flow regime. It is also suggested here that either of the first two scenarios may act in the interaction with third scenario.

\section{CONCLUSIONS}

Three cold-season blocking events in the Southern Hemisphere over the Pacific Ocean were studied on the basis of the reanalysis data and potential vorticity as a diagnostic tool. Phase diagrams were used to find the connection between the decay of these blocks and changes in the planetary-scale flow regimes. In addition, stability of different regimes was estimated by means of calculating the Lyapunov exponents for a diagnosis of the processes linked to blocks.

The results obtained from this analysis indicate that phase diagrams can be used to study the dynamics of blocking regimes. As can be judged from the behavior of Southern Hemisphere blocking events, the decay periods of blocks are consistent with changes in the planetary-scale flow regimes. On the basis of $[1,16,17]$, it can be concluded that the large-scale flow provides a favorable background for long-lived blocks.

Finally, four different scenarios of block decay were identified: decay with a lack of synoptic support $[3,4,8,9]$, decay with an active role of synoptic processes $[7,9,19]$, or either of these mechanisms acting simultaneously with a sharp change in the character of the planetary-scale flow.

\section{ACKNOWLEDGMENTS}

We would like to thank V.P. Dymnikov for his valuable comments and discussion of these results.

\section{REFERENCES}

1. C. H. Tsou and P. J. Smith, "The Role of Synoptic/Planetary-Scale Interactions during the Development of a Blocking Anticyclone," Tellus A 42, 174-193 (1990).

2. M. S. Tracton, "Predictability and Its Relationship to Scale Interaction Processes in Blocking," Mon. Weather Rev. 118, 1666-1695 (1990).

3. A. R. Lupo and P. J. Smith, "Planetary and SynopticScale Interactions during the Life Cycle of a Mid-Latitude Blocking Anticyclone over the North Atlantic," Tellus A 47, 575-596 (1995).

4. A. R. Lupo, "A Diagnosis of Two Blocking Events That Occurred Simultaneously over the Mid-Latitude Northern Hemisphere," Mon. Weather Rev. 125, 1801-1823 (1997).

5. R. F. C. Marques and V. B. Rao, "A Diagnosis of a LongLasting Blocking Event over the Southeast Pacific Ocean," Mon. Weather Rev. 127, 1761-1776 (1999).

6. S. J. Colucci, "Planetary-Scale Preconditioning for the Onset of Blocking," J. Atmos. Sci. 58, 933-942 (2001).

7. J. P. Burkhardt and A. R. Lupo, "The Planetary and Synoptic-Scale Interactions in a Southeast Pacific Blocking Episode Using PV Diagnostics," J. Atmos. Sci. 62, 1901-1916 (2005).

8. A. R. Lupo, A. R. Kunz, and J. P. Burkhardt, "Planetary and Synoptic Scale Interactions in Southeast Pacific Blocking Using Potential Vorticity Diagnostics: More Evidence for the Paucity of Wave-Wave Interactions in Southern Hemisphere Blocking," in Proceedings of the 21 st Conference on Weather Analysis and Forecast- 
ing/17th Conference on Numerical Weather Prediction (Washington DC, 2005).

9. A. R. Lupo, I. I. Mokhov, S. Dostoglou, et al., "The Impact of the Planetary Scale on the Decay of Blocking and the Use of Phase Diagrams and Lyapunov Exponents As a Diagnostic," in Proceedings of the 21 st Conference on Weather Analysis and Forecasting/17th Conference on Numerical Weather Prediction (Washington DC, 2005).

10. V. P. Dymnikov and A. N. Filatov, Stability of LargeScale Atmospheric Processes (Gidrometeoizdat, Leningrad, 1990) [in Russian].

11. E. Kalnay and L. O. Merkine, "A Simple Mechanism for Blocking," J. Atmos. Sci. 38, 2077-2091 (1981).

12. J. S. Frederiksen, "A Unified Three-Dimensional Instability Theory of the Onset of Blocking and Cyclogenesis," J. Atmos. Sci. 39, 969-982 (1982).

13. G. J. Shutts, "The Propagation of Eddies in Diffluent Jet Streams: Eddy Vorticity Forcing of Blocking Flow Fields," Q. J. R. Meteorol. Soc. 109, 737-761 (1983).

14. S. L. Mullen, "The Local Balances of Vorticity and Heat for Blocking Anticylcones in a Spectral General Circulation Model," J. Atmos. Sci. 43, 1406-1441 (1986).

15. S. L. Mullen, "Transient Eddy Forcing and Blocking Flows," J. Atmos. Sci. 44, 3-22 (1987).

16. K. Haines and A. J. Holland, "Vacillation Cycles and Blocking in a Channel," Q. J. R. Meteorol. Soc. 124, 873-897 (1998).

17. S. J. Colucci and D. P. Baumhefner, "Numerical Prediction of the Onset of Blocking: A Case Study with Forecast Ensembles," Mon. Weather Rev. 126, 773-784 (1998).

18. E. Kalnay, et al., "The NCEP/NCAR 40-Year Reanalysis Project," Bull. Am. Meteorol. Soc. 77, 437-471 (1996).

19. A. R. Lupo and L. F. Bosart, "An Analysis of a Relatively Rare Case of Continental Blocking," Q. J. R. Meteorol. Soc. 125, 107-138 (1999).

20. A. R. Lupo and P. J. Smith, "Climatological Features of Blocking Anticyclones in the Northern Hemisphere," Tellus A 47, 439-456 (1995).

21. I. I. Mokhov, "Climate Changes: Analysis of Global Cycles,” Ann. Geophys. 11 (Suppl.), 334 (1993).

22. I. I. Mokhov, Doctoral Dissertation in Mathematics and Physics (IFA RAN, Moscow, 1995).

23. I. I. Mokhov and A. V. Eliseev, "Changes in the Characteristics of the Quasi-Bieenial Oscillation of Zonal Wind and Temperature in the Equatorial Lower Stratosphere," Izv. Akad. Nauk, Fiz. Atm. Okeana 34, 327-336 (1998) [Izv., Atmos. Ocean. Phys. 34, 291-299 (1998)].

24. I. I. Mokhov, A. V. Elieev, and D. V. Khvorost'yanov, "Evolution of the Characteristics of Interannual Climate Variability Associated with the El Niño and La Niña Phenomena," Izv. Akad. Nauk, Fiz. Atm. Okeana 36, 741-751 (2000) [Izv., Atmos. Ocean. Phys. 36, 681-690 (2000)].

25. I. I. Mokhov, D. V. Khvorostyanov, and A. V. Eliseev, "Decadal and Longer-Term Changes in ENSO Characteristics," Int. J. Climatol. 2, 401-414 (2004).

26. A. V. Federov, S. L. Harper, S. G. Philander, et al., "How Predictable Is El Niño?," Bull. Am. Meteorol. Soc. 84, 911-920 (2003).
27. L. Bengtsson, "Numerical Prediction of Atmospheric Blocking-A Case Study," Tellus 33, 19-42 (1981).

28. A. R. Hansen, "Observational Characteristics of Atmospheric Planetary Waves with Bimodal Amplitude Distributions," Adv. Geophys. 29, 101-134 (1986).

29. R. Shapiro, "Smoothing, Filtering, and Boundary Effects," Rev. Geophys. 8, 737-761 (1970).

30. E. N. Lorenz, "Deterministic Nonperiodic Flow," J. Atmos. Sci. 20, 130-141 (1963).

31. J. M. Nese, J. A. Dutton, and R. Wells, "Calculated Attractor Dimensions for Low-Order Spectral Models," J. Atmos. Sci. 44, 1950-1972 (1987).

32. A. R. Hansen and A. Sutera, "Planetary Wave Amplitude Bimodality in the Southern Hemisphere," J. Atmos. Sci. 45, 3771-3783 (1988).

33. I. I. Mokhov, Diagnostics of the Structure of the Climatic System (Gidrometeoizdat, St. Petersburg, 1993) [in Russian].

34. P. Lynch, "Resonant Rossby Wave Triads and the Swinging Spring," Bull. Am. Meteorol. Soc. 84, 605-616 (2003).

35. J. G. Charney and J. G. DeVore, "Multiple Flow Equilibria in the Atmosphere and Blocking," J. Atmos. Sci. 36, 1205-1216 (1979).

36. S. Yoden, "Bifurcation Properties of Quasi-Geostrophic, Barotropic, Low-Order Model with Topography," J. Meteorol. Soc. Jpn. 63, 535-546 (1985).

37. G. Nitsche, C. Kooperberg, and J. M. Wallace, "Is There Evidence of Multiple Equilibria in Planetary-Wave Amplitude?," J. Atmos. Sci. 51, 314-322 (1994).

38. G. M. Agayan and I. I. Mokhov, "Quasi-Stationary FallSeason Regimes of the Atmosphere in the Northern Hemisphere during the PGEP,' Izv. Akad. Nauk SSSR, Fiz. Atmos. Okeana 25, 1150-1156 (1989).

39. V. P. Dymnikov, E. V. Kazantsev, and V. V. Kharin, "Information Entropy and Local Lyapunov Exponents of a Barotropic Atmospheric Circulation,' Izv. Akad. Nauk, Fiz. Atm. Okeana 28, 425-432 (1992).

40. D. Ruelle, "Characteristic Exponents and Invariant Manifolds in Hilbert Space," Ann. Math. 115, 243-290 (1982).

41. P. Constantin, C. Foias, and R. Temam, "Attractors Representing Turbulent Flows," Mem. Am. Math. Soc. 53 (1985)

42. V. P. Dymnikov and E. V. Kazantsev, "Structure of an Attractor Generated by the System of Equations of a Barotropic Atmosphere," Izv. Akad. Nauk, Fiz. Atm. Okeana 29, 581-595 (1993).

43. J. M. Wiedenmann, A. R. Lupo, I. I. Mokhov, and E. A. Tikhonova, "The Climatology of Blocking Anticyclones of the Northern and Southern Hemispheres: Block Intensity As a Diagnostic," J. Clim. 15 (2002).

44. V. P. Dymnikov and A. N. Filatov, "Some Problems of Mathematical Climate Theory" Izv. Akad. Nauk, Fiz. Atm. Okeana 31, 293-303 (1995).

45. H. Lejenas and H. Okland, "Characteristics of Northern Hemisphere Blocking As Determined from a Long Time Series of Observational Data," Tellus A 35, 350-362 (1983).

Vol. 43 No. $1 \quad 2007$ 